\section{Ab 1. Januar 2002 gilt die Preisanschreibe- pflicht für Arzneimittel}

\author{
M. Giger, A. Gehler, R. Gmür
}

Bekanntlich müssen die Vertriebsfirmen von Arzneimitteln seit dem 1. Januar 2001 die Preise nicht mehr auf der Verpackung anschreiben. Aufgrund der Verordnung vom 11. Dezember 1978 über die Bekanntgabe von Preisen (PBV) ist dies neu Sache des Detailhandels, d.h. der Apotheken, Arztpraxen mit direkter Medikamentenabgabe und Drogerien. Das Staatssekretariat für Wirtschaft (Seco) hat dazu Ende 2001 ein Informationsblatt herausgegeben. Gemäss diesem Informationsblatt muss jedes Arzneimittel mit dem tatsächlich zu bezahlenden Preis in Schweizer Franken angeschrieben sein, unabhängig von der Abgabekategorie (A, B, C, D oder E) und unabhängig von der Abgabestelle (Apotheke, Drogerie, Arztpraxis, Spital). Die Preise für nicht frei zugängliche (d.h. rezeptpflichtige) Arzneimittel müssen an der Ware selbst angebracht werden (Anschrift auf der Packung von Hand, per Etikette, per Maschine). Die Preise für frei zugängliche Arzneimittel können wahlweise auch durch Preisschilder, Regalanschrift oder Anschlagen von Preislisten bekanntgegeben werden.

Korrespondenz:

Ärzte mit Patientenapotheke (APA)

c/o Mediapolis

Bionstrasse 4

CH-9015 St. Gallen

Rechtsdienst FMH

Elfenstrasse 18

CH-3000 Bern 16
Entgegen der von der FMH in der Vernehmlassung vertretenen Haltung ist das Seco überdies - und ohne dies in seinem Informationsblatt zu präzisieren - der Auffassung, der Preis müsse schon vor dem Verkauf auf der Packung angebracht werden. Immerhin müsse nicht das ganze Lager im voraus etikettiert werden, es genüge, die zu erwartende monatliche Verkaufsmenge im voraus zu beschriften ... (sic!).

Es ist fraglich, ob diese Interpretation der SecoMitarbeiter rechtlich überhaupt haltbar ist. Die Preisanschrift macht sicher Sinn für Waren, die dem Konsumenten offen angeboten werden. Sinn der PBVVorschriften ist es nämlich, dass die Konsumenten die Preise bei verschiedenen Anbietern unbehelligt vergleichen können. Auch der sakrosankte Wettbewerb samt PBV wird es dem Patienten allerdings nie erlauben, zwecks Preisvergleich in ärztlichen Arzneimittelschränken oder Schubladen der Apotheken und Arztpraxen ähnlich wie in Buchhandlungen zu stöbern und sich aufgrund dieses Preisvergleichs die Abgabestelle für rezeptpflichtige Arzneimittel auszusuchen.

Auf der anderen Seite hält es die FMH für wichtig, dass die Preise auf den Arzneimitteln stehen. Dies weniger aus Gründen des Preiswettbewerbs, der im staatlich regulierten Arzneimittelbereich ohnehin kaum spielt, sondern zur Förderung des Kostenbewusstseins und damit letztlich auch der Compliance der Patienten. Und dafür genügt es in jedem Fall, den Preis anlässlich der Abgabe, zusammen mit der Einnahmevorschrift, auf die Packung zu schreiben. Diese Haltung, vertreten auch an der Konferenz des Eidgenössischen Departements des Innern (EDI) zur Kostenkontrolle im Medikamentenbereich vom 2. Juli 2001, wurde übrigens auch von der anwesenden Vertreterin der Konsumentenorganisationen geteilt.

Die FMH hat indes immer betont, dass es sich dabei nur um eine Notlösung handeln kann. Es liegt auf der Hand, dass die Preise dort angeschrieben werden sollen, wo es am effizientesten möglich ist, also bei Arzneimitteln wie gehabt auf Industrie- oder Grossistenstufe. Wir sind zuversichtlich, dass kundenfreundliche Firmen die Preisanschrift schon bald (wieder) als Dienstleistung entdecken und wahrnehmen werden. Jeder einzelne tut im eigenen Interesse gut daran, seinen Ärztelieferanten/Grossisten darauf anzusprechen und bei der Wahl des Anbieters dessen Dienstleistungspalette zu berücksichtigen. 